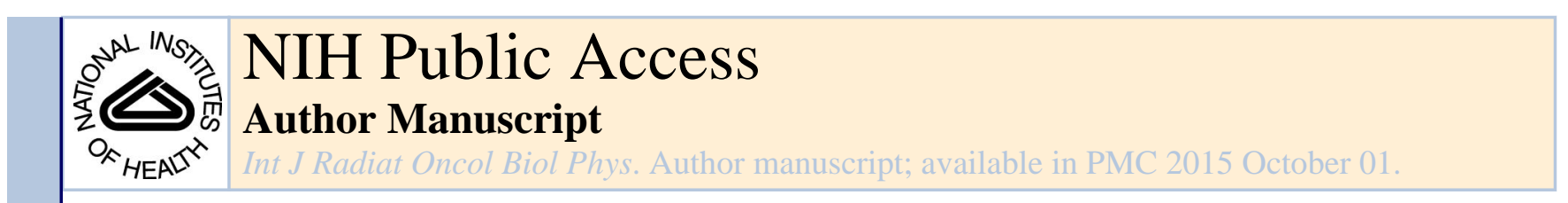

Published in final edited form as:

Int J Radiat Oncol Biol Phys. 2014 October 1; 90(2): 320-328. doi:10.1016/j.ijrobp.2014.06.005.

\title{
Comparison and Consensus Guidelines for Delineation of Clinical Target Volume for CT- and MR-Based Brachytherapy in Locally Advanced Cervical Cancer
}

\author{
Akila N. Viswanathan ${ }^{1}$, Beth Erickson ${ }^{2}$, David K. Gaffney ${ }^{3}$, Walter Bosch ${ }^{4}$, and members of \\ the CT/MR Atlas Committee $1,2,3,4,5,6,7,8,9,10,11,12,13,14,15,16,17,18,19,20$ \\ ${ }^{1}$ Brigham \& Women's Hospital/Dana-Farber Cancer Institute, Boston, MA \\ ${ }^{2}$ Medical College of Wisconsin, Milwaukee, WI \\ ${ }^{3}$ University of Utah Huntsman Cancer Hospital, Salt Lake City, UT \\ ${ }^{4}$ Washington University, St. Louis, MO \\ ${ }^{5}$ University of Pittsburgh Cancer Institute, Pittsburgh, PA \\ ${ }^{6}$ University of lowa, lowa City, IA \\ ${ }^{7}$ University of Alabama, Birmingham, AL \\ ${ }^{8}$ London Health Sciences Centre and Western University, London, ON, Canada \\ ${ }^{9}$ Mayo Medical Center, Rochester, MN \\ 10University of Texas MD Anderson Cancer Center, Houston, TX \\ ${ }^{11}$ University of North Carolina, Chapel Hill, NC \\ ${ }^{12}$ Case Western Reserve University, Cleveland, $\mathrm{OH}$ \\ ${ }^{13}$ University of Pennsylvania, Philadelphia, PA \\ ${ }^{14}$ University of Washington, Seattle, WA \\ ${ }^{15}$ Division of Radiotherapy, Institute of Oncology Ljubljana, Ljubljana, Slovenia \\ ${ }^{16}$ Department of Radiation Oncology, National Center for Cancer Care and Research, Doha, \\ Qatar
}

(C) 2014 Elsevier Inc. All rights reserved.

Address correspondence to: Akila N. Viswanathan, MD, MPH, Brigham and Women's Hospital, Department of Radiation Oncology, 75 Francis Street, ASB 1, L2, Boston, MA 02115, Tel: 617-732-6331, Fax: 617-278-6988, aviswanathan@1roc.harvard.edu. Members of the CT/MR Atlas Committee include:

Sushil Beriwal5, Sudershan K. Bhatia,6, Omer Lee Burnett III,7, David P. D'Souza,8, Nikhilesh Patil,8, Michael G. Haddock,9, Anuja Jhingran,10, Ellen L. Jones,11, Charles A. Kunos,12, Larissa J. Lee,1, Lilie L. Lin,13,Nina A. Mayr,14, Ivy Petersen,9, Primoz Petric,15,,16, Lorraine Portelance,17, William Small Jr.,18, Jonathan B. Strauss,19, Kanokpis Townamchai,1, Aaron H. Wolfson,17 and Catheryn M. Yashar,20

Publisher's Disclaimer: This is a PDF file of an unedited manuscript that has been accepted for publication. As a service to our customers we are providing this early version of the manuscript. The manuscript will undergo copyediting, typesetting, and review of the resulting proof before it is published in its final citable form. Please note that during the production process errors may be discovered which could affect the content, and all legal disclaimers that apply to the journal pertain.

Presented at the annual meeting of the American Society for Radiation Oncology (ASTRO), September, 2013. 
${ }^{17}$ University of Miami Miller School of Medicine, Miami, FL

${ }^{18}$ Loyola University Strich School of Medicine, Chicago, IL

${ }^{19}$ The Robert H. Lurie Comprehensive Cancer Center of Northwestern University, Chicago, IL

${ }^{20}$ University of California, San Diego, CA

\section{Abstract}

Purpose/Objective-To create and compare consensus clinical target volume (CTV) contours for computed tomography (CT) and 3 Tesla (3T) magnetic resonance (MR) image-based cervicalcancer brachytherapy

Materials/Methods-Twenty-three gynecologic radiation oncology experts contoured the same 3 cervical-cancer brachytherapy cases: one Stage IIB near-complete response (CR) case with a tandem and ovoid, one Stage IIB partial response (PR) case with ovoid with needles and one Stage IB2 CR case with a ring applicator. CT contours were completed before MRI contours. These were analyzed for consistency and clarity of target delineation using an expectation maximization algorithm for simultaneous truth and performance level estimation (STAPLE), with kappa statistics as a measure of agreement between participants. The conformity index (CI) was calculated for each of the six data sets. Dice coefficients were generated to compare CT and MR contours of the same case.

Results-For all 3 cases, the mean tumor volume was smaller on MR than on CT ( $\mathrm{p}<0.001)$. Kappa and CI estimates were slightly higher for CT, indicating a higher level of agreement on CT. DICE coefficients were $89 \%$ for the Stage IB2 case with a CR, $74 \%$ for the Stage IIB case with a PR, and $57 \%$ for the Stage IIB case with a CR.

Conclusion-When comparing MR- to CT-contoured CTV volumes, the higher level of agreement on CT may be due to the more distinct contrast visible on the images at the time of brachytherapy. The largest difference at the time of brachytherapy was in the case with parametrial extension at diagnosis that had a near-complete response, due to the appearance of the parametria on CT but not on MR. Based on these results, a 95\% consensus volume was generated for $\mathrm{CT}$ and for MR. Online contouring atlases are available for instruction at http:// www.nrgoncology.org/Resources/ContouringAtlases.aspx.

\section{Keywords}

Radiation; brachytherapy; CT; MR; cervical cancer

\section{INTRODUCTION}

Women with locally advanced cervical cancer require treatment with external beam radiation (EBRT) combined with brachytherapy (BT) in order to maximize both local and regional tumor control. ${ }^{1}$ Survival rates decrease significantly for patients who cannot receive BT to the primary tumor for various reasons. ${ }^{2,3}$ BT requires that a tandem be inserted into the uterus ${ }^{4}$ to bring the primary cervical tumor to doses in the range of 80-90 Gy, depending on tumor size, with the dose historically recorded at Point A. ${ }^{5}$ Proper applicator placement significantly improves local control and disease-free survival. ${ }^{6}$ The advantages of 3D- 
imaging at the time of BT include assurance of proper applicator placement, more accurate definition and treatment of the tumor volume, and delineation of outlines of the OAR for volume-based dose calculations.

Surveys in the U.S. and Europe demonstrate the increasing use of 3D image-based BT, with dose given to the at-risk volume rather than to a pre-specified point. ${ }^{7,8} 3 \mathrm{D}$ image-based $\mathrm{BT}$ results in outstanding local control and a significant reduction in toxicity for cervical-cancer patients. ${ }^{9-11}$ Over the past decade, research has evaluated real-time $0.5 \mathrm{~T}$ MR guidance ${ }^{12}$ or post-insertion MR-imaging to assist with tumor delineation and tumor dosimetry. MR-based planning ${ }^{13}$ has shown favorable results compared to traditional point A plans. ${ }^{14-16}$ Contouring guidelines have been published by the GEC-ESTRO to aid the physician in contouring on 0.2-1.5T MR in a standardized fashion. ${ }^{17,18} \mathrm{~A}$ report using 3T MR for both intracavitary and interstitial gynecologic BT shows the feasibility of using high-strength $3 \mathrm{~T}$ MR units for gynecologic BT. ${ }^{19}$

Contouring accurately on the 3D imaging obtained after applicator placement is critical to ensure an optimal treatment plan that adequately doses the tumor and minimizes the dose to the normal-tissue structures. However, no consensus atlas is available in the U.S. to teach those transitioning from 2D film-based to 3D image-based BT. Prospective clinical trials in cervical cancer encourage image-based BT, and an atlas will be useful for clinical protocols and routine practice.

With the increasing availability of CT simulators in Radiation Oncology departments, CT imaging after BT applicator insertion is easy to implement. MR imaging is feasible but more difficult for most clinics given the typical location of MR scanners outside of the Radiation Oncology department. CT/MR-compatible tandem-and-ring and tandem-and-ovoid applicators are widely available, with or without the addition of interstitial needles. CTcontouring guidelines were generated in a previous comparison study to MRI; the contours on CT were consistently wider in the lateral direction. ${ }^{20} \mathrm{~A} \mathrm{CT}$ scan can define a clinical tumor volume (CTV) around the lateral borders of the cervix and include any obvious parametrial extension seen on the scan. ${ }^{20}$ Uterosacral ligaments may be clearly visualized on $\mathrm{CT}$ when involved with tumor and included in the CTV contours. However, the gross tumor volume (GTV) may not be adequately delineated on CT due to difficulty in identifying the tumor consistently even with IV contrast. Furthermore, the superior border of the cervix is not well visualized on CT, but rather the entire tandem length is activated and the top dwell is optimized to reduce sigmoid and small bowel dose. In order to create a safety margin around the visualized volume, both $\mathrm{CT}^{20}$ and $\mathrm{MR}^{18} \mathrm{HR}-\mathrm{CTV}$ contouring recommendations stated that the parametrial tissues should be included when involved at diagnosis.

The aim of this study was to compare the contours achieved by a large group of expert physicians on CT- and MR-based BT cases representing 3 commonly seen scenarios, and to generate consensus contours for CT and MR brachytherapy atlases to be available on the Web for online training of physicians. 


\section{MATERIALS AND METHODS}

Representative physicians from the RTOG Gynecologic Cancer Working Group and other gynecologic cancer experts received the same 3 cervical-cancer cases as DICOM files which could be uploaded into their institutions' contouring software. Physicians responded to a questionnaire whether they used MR, CT and/or plain film for the majority of their cases. Each case had a 3T MRI at diagnosis, an MRI and a CT performed at the time of BT (within an hour of applicator insertion), and clinical drawings based on the exam at diagnosis and at the time of BT available for physician review. The T2-weighted images were used for contouring. A CT (120kVP, 200mA, 30-cm field of view, 1.25-mm slice thickness) at the time of brachytherapy was performed without intravenous contrast for all 3 cases. A previous contouring study generated a normal-tissue atlas and therefore contouring of the organs at risk (OAR) was not required. ${ }^{21}$

\section{Case descriptions}

Case 1 was that of a patient with a Stage IIB bulky adenocarcinoma of the cervix. The tumor involved both the full length of the uterus and the cervix with bilateral parametrial involvement. At the time of BT, she had a near-complete response to EBRT. Tandem and ovoid applicator was inserted. In order to treat the full length of the tumor, a tandem was inserted to the top of the fundus. Due to the presence of tumor, the tandem extended slightly through the tip of the fundus as was noted on CT, indicating a perforation. In general, perforations of the uterus most commonly occur in the posterior myometrium and require immediate repositioning into the uterine canal and antibiotics, followed by treatment. In this case, the tandem was in the uterine canal and only required slight inferior retraction prior to planning and treatment, in addition to antibiotics. The doses were optimized to minimize dose to the adjacent rectum and sigmoid.

Case 2 was that of a patient with a large Stage IIB cervical cancer with a poor response and difficult anatomy after EBRT. A cavity formed in the region where the cervical tumor had originally filled the vault and this area was stiff and noncompliant. Given the large amount of residual tumor remaining after EBRT, a decision was made to insert a tandem and ovoids with the addition of needles through the ovoids. An air gap was inevitable between the cervix and the ovoids, even though small ovoids were inserted, given the stiffness of the tissue and the cavitary formation of the upper vault. Therefore, it was thought that needles could provide dose where a gap existed with the ovoids.

Case 3 was that of a patient with a Stage IB2 cervical cancer that was bulky at diagnosis with a complete response to chemoradiation.

\section{CT-based contouring}

Instructions mandated that $\mathrm{CT}$ contouring be done first without viewing the MRI scan done at the time of BT. Clinical drawings of the disease at diagnosis, disease extension at the time of BT and the MRI scan done at diagnosis could be viewed for CT-based contouring. For CT contouring, physicians drew a CTV-cervix that included the cervix and any notable parametrial extension at the time of BT, but not the entire parametrial region if not involved, 
similar to the HR-CTV for MRI. The cervix contours started at the level of the applicator. Modification of the previously published CT-based guidelines did not mandate setting a parametrial edge other than what was perceived on the scan. On the axial CT, borders were set as follows:

1. Inferiorly at the level of the ring, contour tissue inside the central ring. For ovoids, contour tissue to the level of the ovoids. Add vaginal tissue adjacent to the ring if involved at the time of BT.

2. Superiorly, contour superiorly to the level where the uterus indents (internal os); draw the next $1 \mathrm{~cm}$ as a pointed shape (cone). The approximate dimension (height) of cervix should be $3 \mathrm{~cm}$.

3. Laterally, parametrial extension should be included in the CT-CTV (and not a separate structure) if it appears "grey/white" on the CT (i.e., a similar density to the cervix). There is no need to draw the parametrial region if it does not have stranding visible on the $\mathrm{CT}$ or it is not noted in the clinical drawing. IV Contrast was not mandated.

\section{MR-based contouring}

The MR-defined-CTV (MR-CTV), which was identical to the GEC-ESTRO defined HR$\mathrm{CTV},{ }^{18}$ included the entire cervix as seen on MR plus any parametrial or vaginal extension (called 'gray zones' by the GEC-ESTRO nomenclature on a low-Tesla MR scan ${ }^{22}$ seen on MRI and on clinical examination at the time of BT. ${ }^{17}$ The Steering Committee determined that physicians would not be asked to contour the GTV or other structures as the primary focus was on comparison of CT-CTV to MR-CTV. A comparison of historic versus current $\mathrm{CT}$ and MR contouring guidelines is summarized in Table 1.

\section{Analysis}

DICOM files were sent to the Advanced Technology Consortium (ATC) for analysis. The Computerized Environment for Radiation Research (CERR), an open source MATLAB (The MathWorks, Natick, MA)-based radiation therapy planning analysis tool ${ }^{23}$, was used to analyze the contours and generated the expectation maximization algorithm for simultaneous truth and performance level estimation (STAPLE) contours ${ }^{24,25}$. MIM software (MIM Software, Inc., Cleveland, OH), was used to smooth the edges of any irregularities. For example, a small portion of the contours extended into the applicator and were removed from the CTV volume.

The clinical cases were then analyzed for consistency and clarity of target delineation using STAPLE, with kappa statistics as a measure of agreement between participants. The conformity index (CI), defined as the ratio between the common (mean) and encompassing (union) volume of a given pair of contours was calculated for each of the data sets independently for MR and for CT. STAPLE sensitivity and specificity values were generated. ${ }^{24,25}$

Consensus atlases were packaged for review using the FullAccess software package (Radialogica, St. Louis, MO) and approved by the NRG Oncology Radiotherapy Committee 
prior to posting on the website (www.rtog.org). The software allows viewing of the CT contour alone or in conjunction with the MR contour.

\section{Comparison of MR versus CT}

Dice coefficients were generated to compare CT and MR contours of the same case. The Dice coefficient is the volume of the intersection divided by the average volume of the two structures.

\section{Dosimetric comparison}

Dosimetric calculations of the D90 and D2cc to the bladder, rectum and sigmoid were performed using both a standard "point A" plan, to a CT- and to an MR-optimized plan. Optimization ensured that the CT or MR CTV D90 was maximized while reducing doses to the organs at risk (OAR), the sigmoid, rectum and bladder as far as was feasible.

\section{RESULTS}

A total of 23 physicians contoured as part of this protocol. A total of 50\% report using MR for brachytherapy contouring routinely; $90 \%$ use CT for brachytherapy planning either in addition or instead of MR routinely. The mean, minimum and maximum tumor volumes for each of the 3 cases on CT and MR are listed in Table 2. The mean tumor volume was smaller on MR than on CT for all 3 cases $(\mathrm{p}<0.001)$. Sensitivity and specificity were similar between CT and MR (Table 1), indicating very little apparent difference in contours. Kappa estimates showed substantial agreement among physicians' contours and were significantly higher for CT ( $\mathrm{p}=0.048)$, with a mean value on CT of 0.69 versus 0.66 on MR. Conformity index was significantly higher for CT compared to MR ( $\mathrm{p}=0.048)$, indicating a higher level of agreement on CT. Dice coefficients of the $95 \%$ consensus volumes comparing CT to MR were $57 \%$ for case $1,74 \%$ for case 2 and $89 \%$ for case 3 . A CT atlas and an MR atlas were generated based on the consensus contours for case 1 (Figure 1), case 2 (Figure 2) and case 3 (Figure 3). The individual physician contours on CT and MR are shown for case 2 (Figure 4 ) and case 3 (Figure 5). The highest Dice coefficient was found for case 3, which had a smaller initial tumor with no parametrial involvement, and a small amount of residual disease to contour, with clear edges of the cervix visible on CT and MR (Figure 3). Case 1, a patient with a large tumor at diagnosis with parametrial extension that had a good response to EBRT, had the lowest Dice coefficient due to the appearance of parametrial extension on the CT but not on the MR (Figure 1). Case 2 had a large tumor at diagnosis and large residual disease at the time of BT; the contours for this case on both CT and MR (Figure 2) were large, indicating a good level of concordance between consensus contours in this clinical scenario.

\section{Dosimetric analysis}

Despite a consistently larger volume on CT for all 3 cases, there was no statistically significant difference in D90 or D2cc OAR comparing CT to MR. Case 1 (large tumor, intermediate response) had a large difference between CT and MR volumes and also had the largest difference between MR and CT optimized D90. Table 2 shows the mean DVH values 
for all 3 cases combined However, this did not translate to statistically significant dosimetric differences (all $\mathrm{p}>0.05$ ). Table 2 shows the mean DVH values for all 3 cases combined.

\section{DISCUSSION}

This study analyzed detailed contouring from a large group of expert gynecologic-cancer radiation oncologists using 3 different types of brachytherapy applicators to generate CTand 3T MR-based cervical-cancer brachytherapy atlases. These atlases can be used in future cervical-cancer trials and clinical practice. In the three different clinical scenarios analyzed, the CT-generated CTV contours were more similar among physicians than were the MRbased contours, indicating a higher degree of reliability. Both CT and MR had high sensitivity and specificity. There was greater discordance between CT and MR in cases with parametrial extension and a good response to EBRT than in either cases with no parametrial extension and a small tumor with a good response to EBRT or cases with parametrial extension and a poor response to EBRT. The comparison between MR and CT validates earlier findings showing that the MR-contoured volume was consistently smaller than the CT-contoured CTV, ${ }^{20}$ and clarifies that this discrepancy is greatest in patients who have parametrial extension at diagnosis and a good response to treatment. Case 1, a patient with a large tumor at diagnosis with parametrial extension that had a good response to EBRT, had the lowest Dice coefficient due to the appearance of parametrial extension on the CT but not on the MR. CT may overestimate volume in patients with parametrial extension at diagnosis that have a good response to EBRT. Conversely, it is possible that with 3T MR, parametrial regions that were initially involved may no longer enhance due to scar tissue, particularly in patients that have had a complete clinical response. Whether this lack of enhancement means that micrometastatic residual disease may still be present is not known. The incidence of parametrial recurrence in patients who have had a good response on 3T MR but have residual parametrial extension on $\mathrm{CT}$ must be analyzed by large prospective series.

Accurate delineation of the tumor and organs at risk is critical for optimal treatment planning. Due to the rapid fall-off of dose, imprecise contouring can dramatically change dosing to the tumor and to the adjacent normal tissue. In this study, the CIs (ratio between common and encompassing volumes) of a large number of gynecologic experts were between 0.37-0.48. For each clinical scenario, the CI was slightly higher for CT than for MR, likely due to the distinction on CT between involved 'grey' region and noninvolvement of the parametria. All 3 cases had 3T MRI, which provides excellent soft-tissue resolution.

In a study analyzing the agreement between target volumes, as delineated by two observers on two different MR image planes, the inter-observer and inter-planar CIs were ranged from $0.7-0.8,{ }^{26}$ with small volumetric and dosimetric variations. ${ }^{27}$ The CIs in our analysis were lower due to the complexity of the cases and the large number of expert physicians contouring, though all had significant experience contouring on both MR and CT-based implants.

In a report of 6 cases contoured by 10 physicians, results showed that, due to lower delineation uncertainties when compared to GTV and IR-CTV, the HR-CTV may be 
considered the most robust volume for dose prescription and optimization. ${ }^{28}$ In that study, no comparison to CT was made. The dosimetric consequence on MR CTV was a single fraction mean relative standard deviation of $8-10 \%$ and a cumulative whole-treatment uncertainty of $+/-5 \mathrm{~Gy} .{ }^{29}$ Similarly, in this study, we chose to only focus on the MR-CTV on MR and the CT-CTV on CT.

The DICE coefficient of the $95 \%$ consensus volumes comparing CT to MR was highest (89\%) for the case with no parametrial extension and a good response to EBRT, the scenario most often taught in contouring workshops. These cases are therefore the least likely to benefit from the use of MR. In contrast, the scenario with a large tumor volume at diagnosis that had a near complete response had the lowest DICE coefficient (57\%), with significant differences between CT and MR contours in the region of the parametria (Figure 1). In this scenario, caution must be exerted when using MR to contour the CTV in that even amongst expert physicians, the parametria may appear to have a complete response, whereas on CT, this region appears as scar and is treated to full dose as part of the CTV. Whether MR may have a higher rate of parametrial failure as a consequence of this potential undercontouring, or whether CT may overdose the parametria and/or organs at risk in this scenario is unknown. In order to safely treat all patients presenting with this scenario, caution in covering the full parametria is recommended at this time. The limitations of intracavitary techniques in the setting of bulky parametrial involvement, however, must be realized, and when appropriate, interstitial techniques may need to be considered.

In the scenario with a large tumor that had an incomplete response, an applicator that incorporates interstitial needles into the ovoids was utilized. This scenario resulted in a DICE coefficient of $74 \%$ comparing CT to MR consensus volumes. The highest discrepancy was again in the parametrial region, but more parametrial tissue was contoured on the MR compared to case 1, resulting in the higher DICE coefficient. This reiterates the importance of careful evaluation of the parametria on MR. For centers where only a CT is available, the CT suffices to cover adequate parametrial extension in all scenarios if the contours extend to the most lateral aspect of the parametrial tissue.

Based on these three scenarios, parametrial extension may help clarify when patients may benefit from an MRI at the time of brachytherapy. In cases with no parametrial extension, MR and CT have nearly identical CTV contours. For cases with parametrial extension and a poor response to treatment, MR and CT have similar CTV contours. Cases with parametrial extension with a complete response benefit the most from utilizing both MRI and CT. Table 2 summarizes the current recommendations for CT -based contouring.

A direct comparison of CT to $0.2 \mathrm{~T} \mathrm{MR}$ planning after insertion in the same patient showed that the CT-contoured images were wider in the region of the parametria. ${ }^{20}$ The first prospective study using mainly CT-based planning in comparison to plain X-ray-based planning, the French STIC trial, showed significant improvements with CT in the subgroup receiving chemo-radiation for locally advanced disease in toxicity reduction (22\% to $2.6 \%$ ), and not significant improvements in local control (74\% to $79 \%)$, disease-specific survival (55\% to $60 \%$ ) and overall survival (65\% to $74 \%) .{ }^{9}$ Other institutional series using $\mathrm{CT}^{30,31}$ 
confirm that very high rates of local control ( $90 \%$ and above) are achievable with limited rates of grade 3 or higher morbidity.

\section{Conclusion}

To date, no other study has compared consensus contours generated by a large group of expert gynecologic radiation oncologists using $\mathrm{CT}$ and $3 \mathrm{~T}$ MR. MRI-contoured volumes are smaller than CT volumes, particularly in cases with parametrial extension, and depend on the amount of tumor regression. CT has a higher level of agreement that may be due to the more distinct contrast between tissues on the images at the time of brachytherapy. A $95 \%$ consensus volume was generated for $\mathrm{CT}$ and for MR online contouring atlases available for instruction at http://www.nrgoncology.org/Resources/ContouringAtlases.aspx based on these results.

\section{Acknowledgments}

Financial disclosures: Dr. Viswanathan receives support through NIH R21 167800. The Advanced Technology Consortium (ATC) has received support through NIH Grant CA81647.

\section{REFERENCES}

1. NCCN. Guidelines for Cervical Cancer. In. 2013

2. Montana GS, Hanlon AL, Brickner TJ, et al. Carcinoma of the cervix: patterns of care studies: review of 1978, 1983, and 1988-1989 surveys. Int J Radiat Oncol Biol Phys. 1995; 32:1481-1486. [PubMed: 7635793]

3. Lanciano RM, Martz K, Coia LR, Hanks GE. Tumor and treatment factors improving outcome in stage III-B cervix cancer. International Journal of Radiation Oncology Biology and Physics. 1991; 20:95-100.

4. Viswanathan AN, Cormack R, Rawal B, Lee H. Increasing brachytherapy dose predicts survival for interstitial and tandem-based radiation for stage IIIB cervical cancer. Int J Gynecol Cancer. 2009; 19:1402-1406. [PubMed: 20009897]

5. Viswanathan AN, Thomadsen B. American Brachytherapy Society consensus guidelines for locally advanced carcinoma of the cervix. Part I: general principles. Brachytherapy. 2012; 11:33-46. [PubMed: 22265436]

6. Viswanathan AN, Moughan J, Small W Jr, et al. The quality of cervical cancer brachytherapy implantation and the impact on local recurrence and disease-free survival in radiation therapy oncology group prospective trials 0116 and 0128. Int J Gynecol Cancer. 2012; 22:123-131. [PubMed: 22193645]

7. Viswanathan AN, Erickson BA. Three-dimensional imaging in gynecologic brachytherapy: a survey of the American Brachytherapy Society. Int J Radiat Oncol Biol Phys. 2010; 76:104-109. [PubMed: 19619956]

8. Viswanathan AN, Creutzberg CL, Craighead P, et al. International brachytherapy practice patterns: a survey of the Gynecologic Cancer Intergroup (GCIG). Int J Radiat Oncol Biol Phys. 2012; 82:250-255. [PubMed: 21183288]

9. Charra-Brunaud C, Harter V, Delannes M, et al. tImpact of 3D image-based PDR brachytherapy on outcome of patients treated for cervix carcinoma in France: Results of the national STIC prospective study. Radiother Oncol. 2012 Epub.

10. Potter R, Georg P, Dimopoulos JC, et al. Clinical outcome of protocol based image (MRI) guided adaptive brachytherapy combined with 3D conformal radiotherapy with or without chemotherapy in patients with locally advanced cervical cancer. Radiother Oncol. 2011; 100:116-123. [PubMed: 21821305] 
11. Haie-Meder C, Chargari C, Rey A, Dumas I, Morice P, Magne N. MRI-based low dose-rate brachytherapy experience in locally advanced cervical cancer patients initially treated by concomitant chemoradiotherapy. Radiother Oncol. 2010; 96:161-165. [PubMed: 20655120]

12. Viswanathan AN, Szymonifka J, Tempany-Afdhal CM, O'Farrell DA, Cormack RA. A prospective trial of real-time magnetic resonance-guided catheter placement in interstitial gynecologic brachytherapy. Brachytherapy. 2013; 12:240-247. [PubMed: 23415048]

13. Kirisits C, Potter R, Lang S, Dimopoulos J, Wachter-Gerstner N, Georg D. Dose and volume parameters for MRI-based treatment planning in intracavitary brachytherapy for cervical cancer. Int J Radiat Oncol Biol Phys. 2005; 62:901-911. [PubMed: 15936576]

14. Lindegaard JC, Tanderup K, Nielsen SK, Haack S, Gelineck J. MRI-guided 3D optimization significantly improves DVH parameters of pulsed-dose-rate brachytherapy in locally advanced cervical cancer. Int J Radiat Oncol Biol Phys. 2008; 71:756-764. [PubMed: 18191335]

15. Jurgenliemk-Schulz IM, Tersteeg RJ, Roesink JM, et al. MRI-guided treatment-planning optimisation in intracavitary or combined intracavitary/interstitial PDR brachytherapy using tandem ovoid applicators in locally advanced cervical cancer. Radiother Oncol. 2009; 93:322-330. [PubMed: 19748695]

16. De Brabandere M, Mousa AG, Nulens A, Swinnen A, Van Limbergen E. Potential of dose optimisation in MRI-based PDR brachytherapy of cervix carcinoma. Radiother Oncol. 2008; 88:217-226. [PubMed: 18037524]

17. Potter R, Haie-Meder C, Van Limbergen E, et al. Recommendations from gynaecological (GYN) GEC ESTRO working group (II): concepts and terms in 3D image-based treatment planning in cervix cancer brachytherapy-3D dose volume parameters and aspects of 3D image-based anatomy, radiation physics, radiobiology. Radiother Oncol. 2006; 78:67-77. [PubMed: 16403584]

18. Haie-Meder C, Potter R, Van Limbergen E, et al. Recommendations from Gynaecological (GYN) GEC-ESTRO Working Group (I): concepts and terms in 3D image based 3D treatment planning in cervix cancer brachytherapy with emphasis on MRI assessment of GTV and CTV. Radiother Oncol. 2005; 74:235-245. [PubMed: 15763303]

19. Kapur T, Egger J, Damato A, Schmidt EJ, Viswanathan AN. 3-T MR-guided brachytherapy for gynecologic malignancies. Magn Reson Imaging. 2012; 30:1279-1290. [PubMed: 22898699]

20. Viswanathan AN, Dimopoulos J, Kirisits C, Berger D, Potter R. Computed tomography versus magnetic resonance imaging-based contouring in cervical cancer brachytherapy: results of a prospective trial and preliminary guidelines for standardized contours. Int J Radiat Oncol Biol Phys. 2007; 68:491-498. [PubMed: 17331668]

21. Gay HA, Barthold HJ, O'Meara E, et al. Pelvic normal tissue contouring guidelines for radiation therapy: a Radiation Therapy Oncology Group consensus panel atlas. Int J Radiat Oncol Biol Phys. 2012; 83:e353-e362. [PubMed: 22483697]

22. Potter R, Dimopoulos J, Kirisits C, et al. Recommendations for image-based intracavitary brachytherapy of cervix cancer: the GYN GEC ESTRO Working Group point of view: in regard to Nag et al. (Int) J Radiat Oncol Biol Phys. Int J Radiat Oncol Biol Phys. 2005; 2005; 6062:1160 1172. 293-5. author reply 5-6.

23. Deasy JO, Blanco AI, Clark VH. CERR: a computational environment for radiotherapy research. Medical physics. 2003; 30:979-985. [PubMed: 12773007]

24. Warfield SK, Zou KH, Wells WM. Simultaneous truth and performance level estimation (STAPLE): an algorithm for the validation of image segmentation. IEEE transactions on medical imaging. 2004; 23:903-921. [PubMed: 15250643]

25. Allozi R, Li XA, White J, et al. Tools for consensus analysis of experts' contours for radiotherapy structure definitions. Radiother Oncol. 2010; 97:572-578. [PubMed: 20708285]

26. Petric P, Dimopoulos J, Kirisits C, Berger D, Hudej R, Potter R. Inter- and intraobserver variation in HR-CTV contouring: Intercomparison of transverse and paratransverse image orientation in 3D-MRI assisted cervix cancer brachytherapy. Radiother Oncol. 2008

27. Dimopoulos JC, De Vos V, Berger D, et al. Inter-observer comparison of target delineation for MRI-assisted cervical cancer brachytherapy: application of the GYN GEC-ESTRO recommendations. Radiother Oncol. 2009; 91:166-172. [PubMed: 19062118] 
28. Petric P, Hudej R, Rogelj P, et al. Uncertainties of target volume delineation in MRI guided adaptive brachytherapy of cervix cancer: a multi-institutional study. Radiother Oncol. 2013; 107:6-12. [PubMed: 23453539]

29. Hellebust TP, Tanderup K, Lervag C, et al. Dosimetric impact of interobserver variability in MRIbased delineation for cervical cancer brachytherapy. Radiother Oncol. 2013; 107:13-19. [PubMed: 23462706]

30. Tan LT, Coles CE, Hart C, Tait E. Clinical impact of computed tomography-based image-guided brachytherapy for cervix cancer using the tandem-ring applicator - the Addenbrooke's experience. Clin Oncol (R Coll Radiol). 2009; 21:175-182. [PubMed: 19101130]

31. Kang HC, Shin KH, Park SY, Kim JY. 3D CT-based high-dose-rate brachytherapy for cervical cancer: clinical impact on late rectal bleeding and local control. Radiother Oncol. 2010; 97:507513. [PubMed: 21074881]

32. Haie-Meder C, Potter R, Van Limbergen E. Recommendations from Gynaecological (GYN) GECESTRO Working Group (I): concepts and terms in 3D image based 3D treatment planning in cervix cancer brachytehrapy with emphasis on MRI assessment of GTV and CTV. Radiother Oncol. 2005; 74:235-245. [PubMed: 15763303] 


\section{Summary}

To date, no other study has compared consensus contours generated by a large group of expert gynecologic radiation oncologists using CT and 3T MR. MRI-contoured volumes are smaller than CT volumes, particularly in cases with parametrial extension, and depend on the amount of tumor regression. CT has a higher level of agreement that may be due to the more distinct contrast between tissues on the images at the time of brachytherapy. A 95\% consensus volume was generated for CT and for MR online contouring atlases available for instruction at http://www.nrgoncology.org/Resources/ ContouringAtlases.aspx. based on these results. 


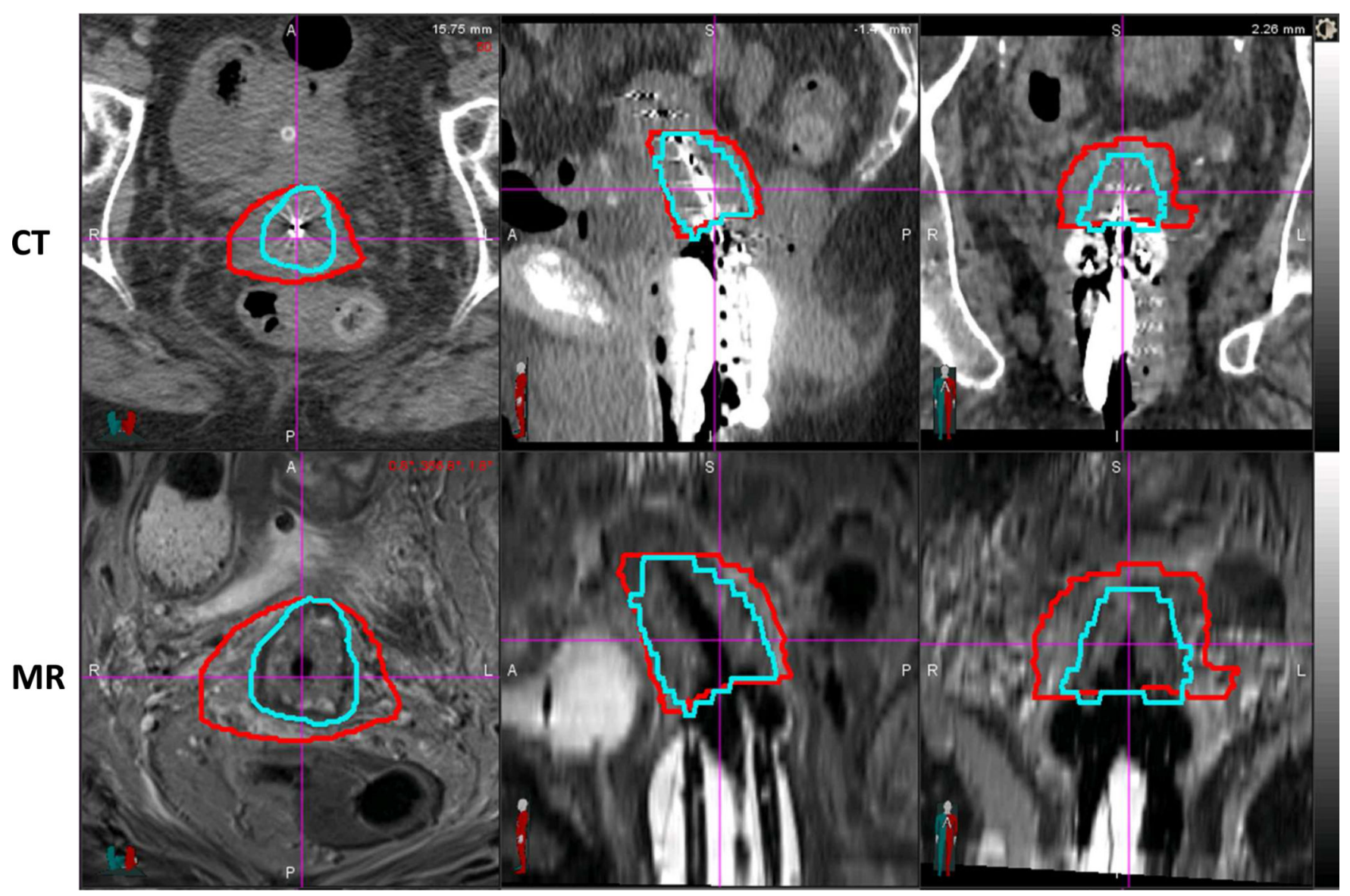

Figure 1.

Axial, sagittal and coronal images of CT and MRI for case 1 showing a tandem and ovoid applicator with consensus contours for MR (light blue) and for CT (red) 


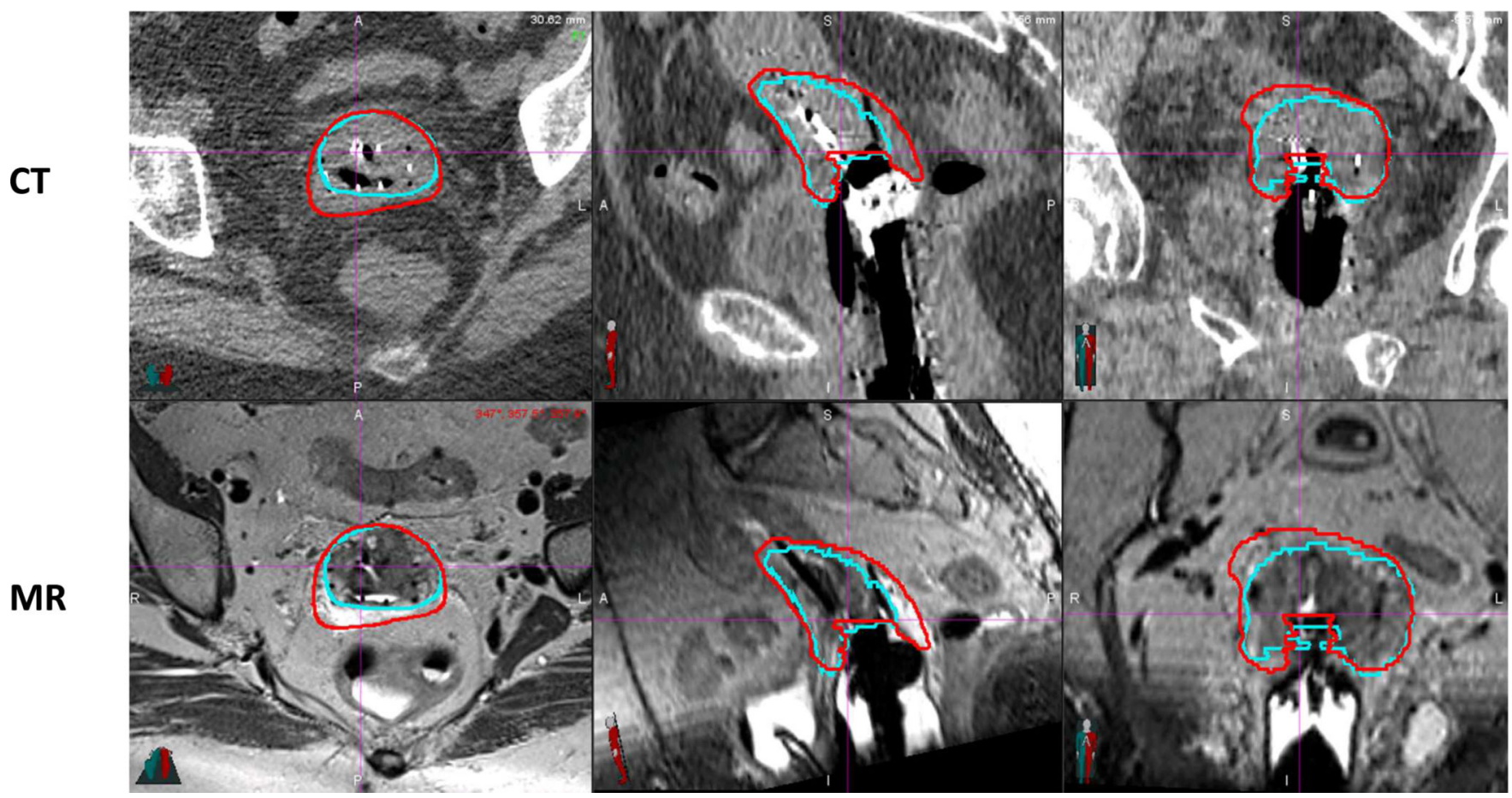

Figure 2.

Axial, sagittal and coronal images of CT and MRI for case 2 showing a tandem and ovoid with needles applicator with consensus contours for MR (light blue) and for CT (red) 


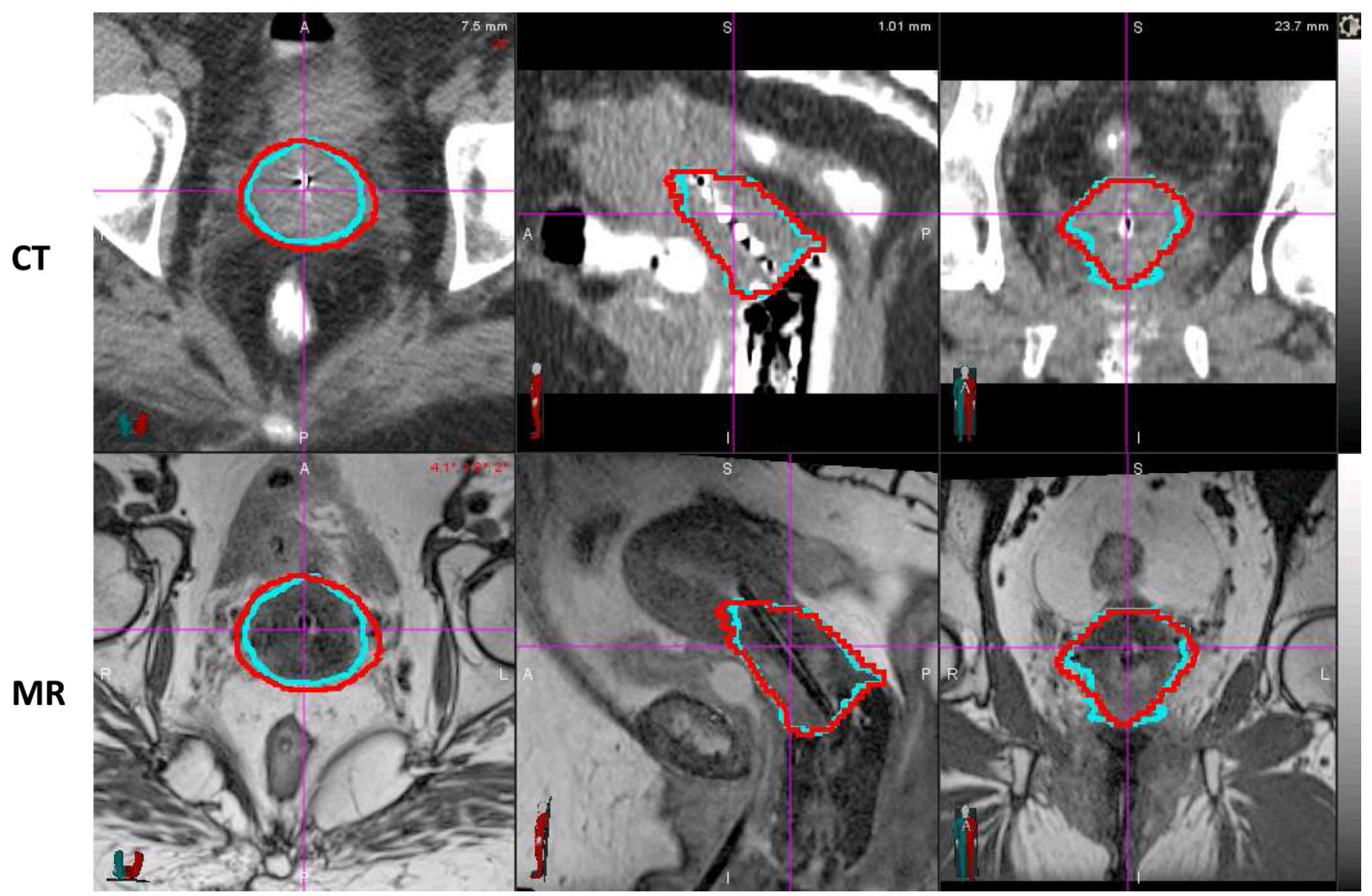

Figure 3.

Axial, sagittal and coronal images of CT and MRI for case 3 showing a tandem and ring applicator with consensus contours for MR (light blue) and for CT (red) 

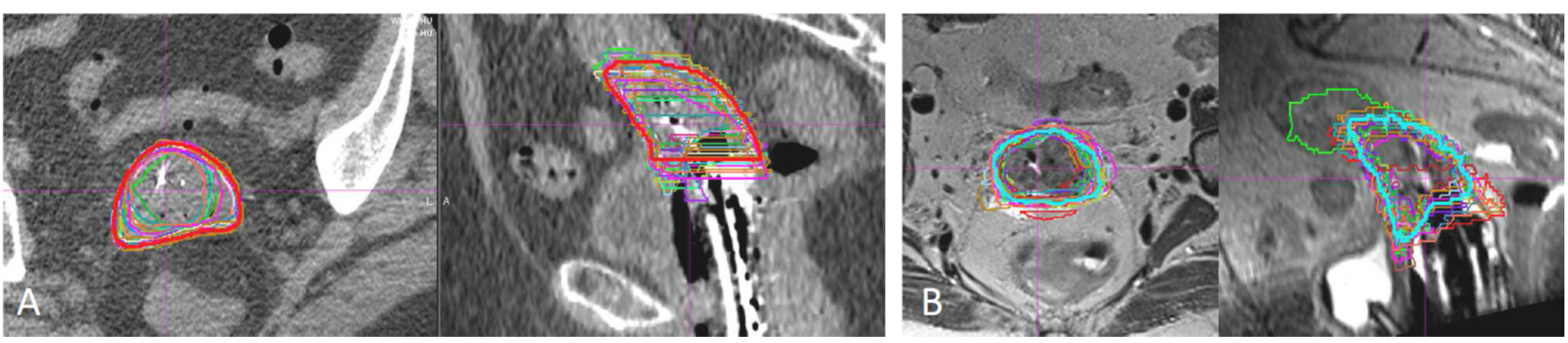

Figure 4. 

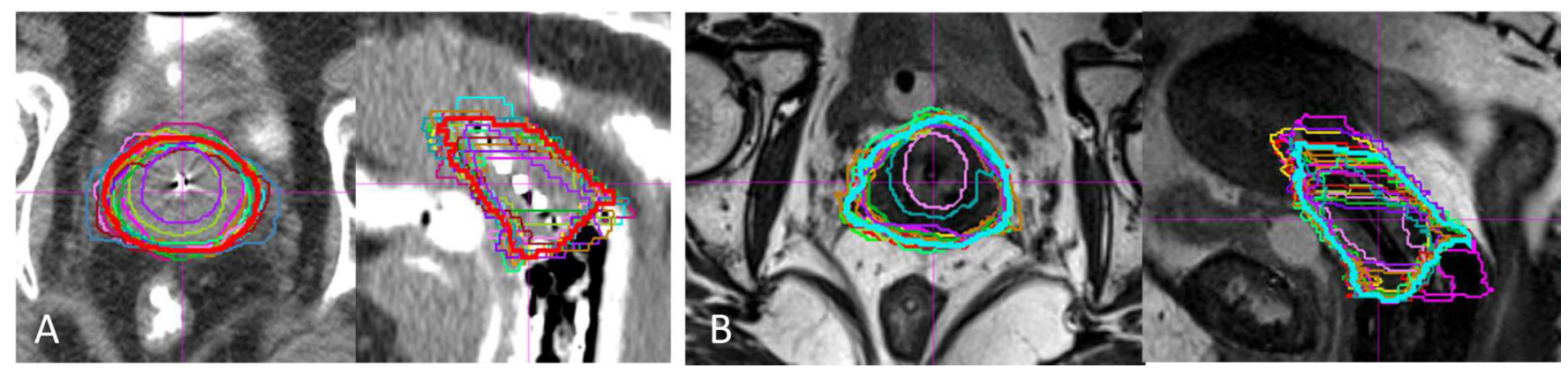

Figure 5. 
Table 1

Comparison of contouring guidelines in locally advanced cervical cancer

\begin{tabular}{|c|c|}
\hline \multicolumn{2}{|c|}{ Clinical Target Volume Contouring Guidelines for MR and CT } \\
\hline MR $(2005)^{32}$ & $\begin{array}{l}\text { Contour the whole cervix and the presumed } \\
\text { extracervical tumor extension at time of BT. Tumor } \\
\text { extension is defined by clinical examination } \\
\text { (visualisation and palpation) and by MRI findings at } \\
\text { time of brachytherapy (BT) taking into account } \\
\text { tumor spread at diagnosis as indicated on clinical } \\
\text { examination and initial MRI for staging. } \\
\text { Pathologic residual tissue(s) as defined by palpable } \\
\text { indurations and/or residual grey zones in } \\
\text { parametria, uterine corpus, vagina or rectum and } \\
\text { bladder on MR are included in HR CTV. No safety } \\
\text { margins are added. }\end{array}$ \\
\hline $\mathrm{CT}(2007)^{20}$ & $\begin{array}{l}\text { Contour entire cervix as seen on CT } \\
\text { 1. Inferiorly, start contour at superior level of applicator. } \\
\text { 2. Superiorly, contour to level at which uterine vessels first abut cervical tissue (if intravenous (IV) contrast administered) } \\
\text { to point at which volume expands (indicating presence of uterine tissue), or to point at which uterine cavity appears. } \\
\text { a. Add two slices of contour (with decreasing diameters) around tandem superiorly to cover conical cervical apex. } \\
\text { b. Measure height of cervix to ensure adequate coverage (average height approximately } 3 \mathrm{~cm} \text { ). Divide parametria into } \\
\text { inner half and outer half. Contour parametria entire height of the cervix. }\end{array}$ \\
\hline $\begin{array}{l}\text { CT (2014, this } \\
\text { manuscript) }\end{array}$ & $\begin{array}{l}\text { 1. Inferiorly at the level of the ring, contour tissue inside the central ring. For ovoids, contour tissue to the level of the } \\
\text { ovoids. Add vaginal tissue adjacent to the ring if involved at the time of BT. } \\
\text { 2. Superiorly, contour superiorly to the level where the uterus indents (internal os); draw the next } 1 \mathrm{~cm} \text { as a pointed shape } \\
\text { (cone). The approximate dimension (height) of cervix should be } 3 \mathrm{~cm} \text {. } \\
\text { 4. Laterally, parametrial extension should be included in the CT-CTV (and not a separate structure) if it appears "grey/ } \\
\text { white" on the CT (i.e., a similar density to the cervix). There is no need to draw the parametrial region if it does not have } \\
\text { stranding visible on the CT or it is not noted in the clinical drawing. IV Contrast is not mandated. } \\
\text { 5. Take into account tumor present on clinical examination and MRI findings at time of BT if available. Disease extension } \\
\text { on clinical exam and MRI at the time of diagnosis should be contoured in a low dose region (Intermediate Risk (IR)- } \\
\text { CTV). } \\
\text { 6. Pathologic residual tissue(s) identified in the uterus, vagina or rectum and bladder are included in the CT-CTV. }\end{array}$ \\
\hline
\end{tabular}




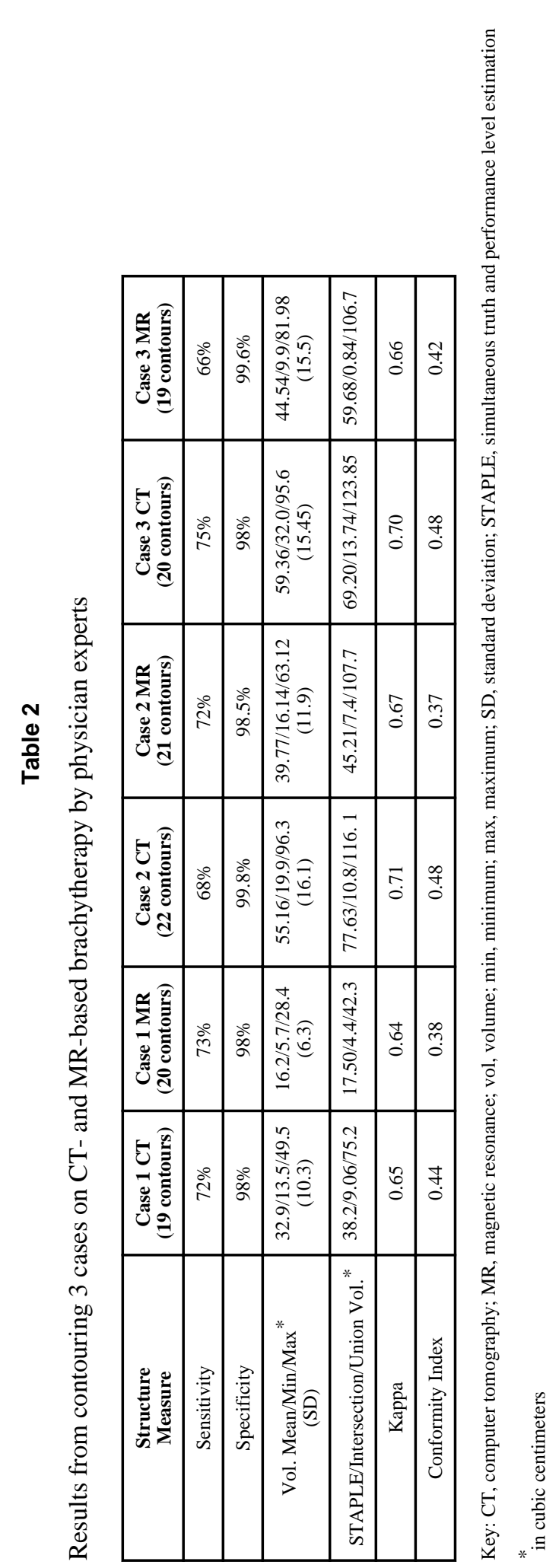

Int J Radiat Oncol Biol Phys. Author manuscript; available in PMC 2015 October 01. 